\title{
ITERATIVE EXCITABILITY IN LILLIE'S NERVE MODEL
}

\author{
KAZUMI YAMAGIWA ${ }^{1}$ \\ Department of Physiology, Tokyo Medico-Dental College, Tokyo
}

Considering the excitability in general as a function of time, Lapicque distinguished the following two cases (1):

A. Dans le cas d l'excitation directe, la sommation, ou l'addition latente, d'épends essentiellemnt de la durèe des stimuli, tandis que:

B. Dans lexcitation indirecte, certaines systèmes ou appareils physiologique, peu ou moins sensibles à un stimulus isolé, quelle que soit la durèe de celui-ci, reagissent dans une large mesure à la réitération plus ou moins rapide, plus ou moins prolongée de ce stimulus long ou bref.

These systems or apparatuses are those which he calls "iterative." The so-called iterative nerve makes a subcategory and contains several nerves intervening between the site of stimulus and the effectors.

Whether it is appropriate or not to classify a certain group of nerves as "iterative" has been questioned (2), but it is not a matter of direct concern in our present problem. We intend, putting this problem aside for the moment, to describe some phenomena observed in modified Lillie's nerve models, in which the stimulus became first effective in producing a certain final event when it was repeated a few times at regular intervals.

\section{METHOD}

A well-polished iron wire was covered with paraffin (fig. 1, $P$ ) for a certain

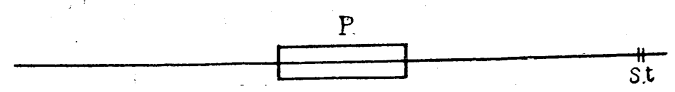

Fig. 1. The model used (schematic). $P$, paraffined part. $S t$, stimulus.

length at about the middle. It was laid in a bath of strong nitric acid to make it passive, and then $\mathrm{Zn}$-stimuli (momentary touches) were applied to one end of it at regular intervals, that is, at every immersion time of certain definite durations, long or short. If, now, the interval is sufficiently long, each activation wave in isolation passes over the $P$-part to travel further, provided that the $P$-part is not longer than a certain limit. But if the interval is short, a number of successive waves stop at $P$ before a transmission takes place. The point of investigation was how many waves were needed for the transmission to occur once, in several rhythms of activation.

The container was about $50 \mathrm{~cm} ., 2.5 \mathrm{~cm}$. and $1 \mathrm{~cm}$. in length, breadth and depth respectively, and the acid was 1.35 in specific gravity and about $60 \mathrm{cc}$.

1 山桠一三

Received for publication October 21, 1950. 
in amount in all experiments performed. As to other details, refer to a previous paper (3).

RESULTS

\section{1. $N($ number $)-I($ interval $)$-relation}

The number of waves in question $(N)$ was found to be in unique relation to the interval $(I)$, that is, the shorter the interval, the more waves were needed. If the interval is too short, then, practically, no transmission occurs.

Remarks. 1) That the transmission evoked by a series of waves was due to theeffect sum mated of each wave, and not due to simple recovery of the post- $P$ region, is clear and certain from the following control examinations : the successive activations were carried out with $K$
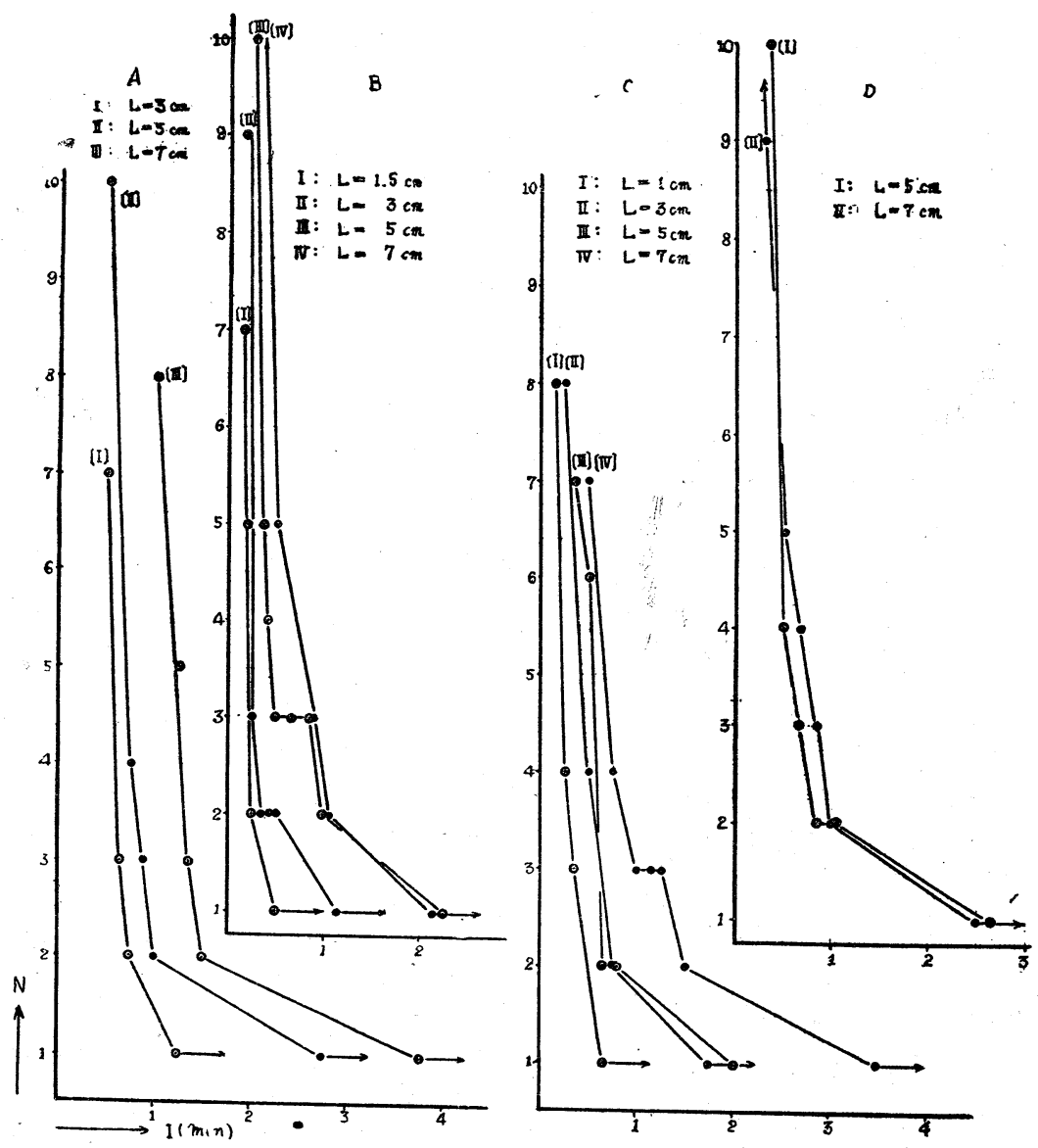

Fig. 2. $N$-I-relation.

$N$, number of waves required for single transmission (ordinates).

$I$, interval of successive activations (abscissa). 
(see fig. 5) open, except the final activation which was done with $K$ closed. The result was that the wave could never pass the $P$-part, although it did when the activations were all made with $K$ closed. 2) Complication sometimes occurred when the interval was so short that each successive wave did not reach the $P$-part but died on the way. In these cases, after a many time repetition of conduction suspension, the on-coming wave was sometimes conducted without any hindrance, and even passed over the $P$-part, evidently due to the summation effect as well as to the sufficient recovery taking place at the region beyond the point of conduction suspension. 3) Another thing to be remarked is that the wave evoked in the post- $P$ region was conducted usually along the whole core, but sometimes it was found to travel just a short distance, when the immersion time or the length of $P$ was minutely controlled. This shows that the system under examination does not always follow the all-or-none principle, though it appears to do so usually. Perhaps it is one of the evidences for the dependence of initiation of propagated wave upon a certain liminal area, the activation of which alters the whole appearance from heterobolic to isobolic. Presumably the area is quite small and accordingly escapes ordinary experimental manipulations.

A few examples of the results obtained are illustrated in fig. 2. If finely measured, there is always found a certain "range" of interval for each $N$, as it is partly visible in fig. $2, B$ and $C$. The whole graph then takes a stair-case shape as illustrated in fig. 3.

\section{NI-I-relation}

From, the $N$-I-relation obtained, the NI-I-relation, that is, the relation between the total time required for a transmission, on one side, and the interval, on the other, was made numerically. The results are illustrated graphically in fig. 4. In many of the curves in fig. 4, except those for small $L$ (the length of the $P$-part), there is a well marked minimum point, expressing that the total time necessary for transmission becomes least when the core is activated at a certain definite rhythm. The lack of such a minimum point, was observed when the $P$-part was relatively short, that is, when the transmission is relatively easy.

If a $N I$-I-curve is constructed from a finely explored stair-case shaped $N$ - $I$-curve, it is shaped as illustrated in fig. 3 , in which the existence of a minimum point appears

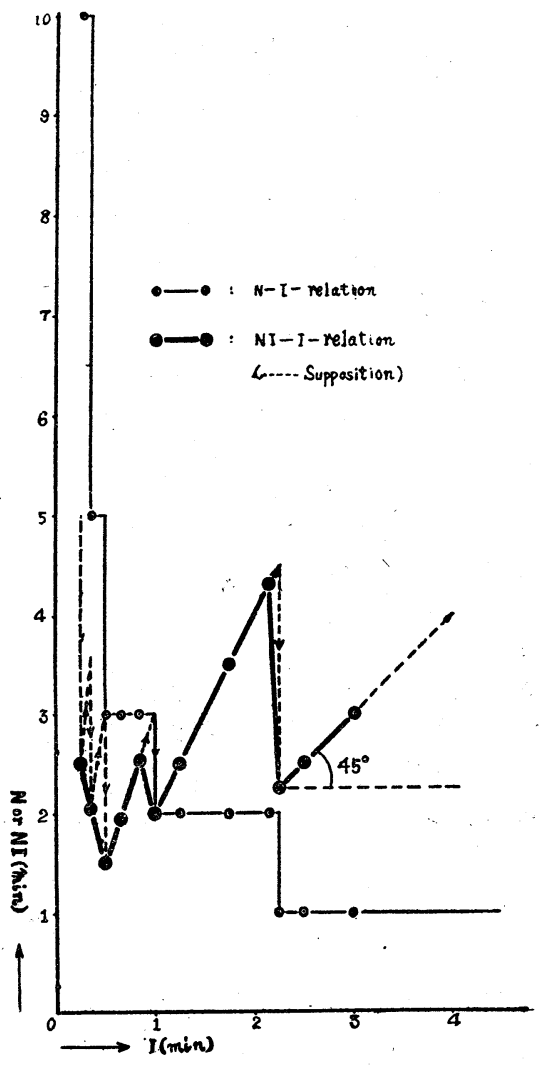

Fig. 3. $N$-I- and NI-I-relations finely explored. much clearer. If, however, the points of the least interval for each $N$ are not finely explored or missing, the minimum point will be hidden or appear only vaguely. 

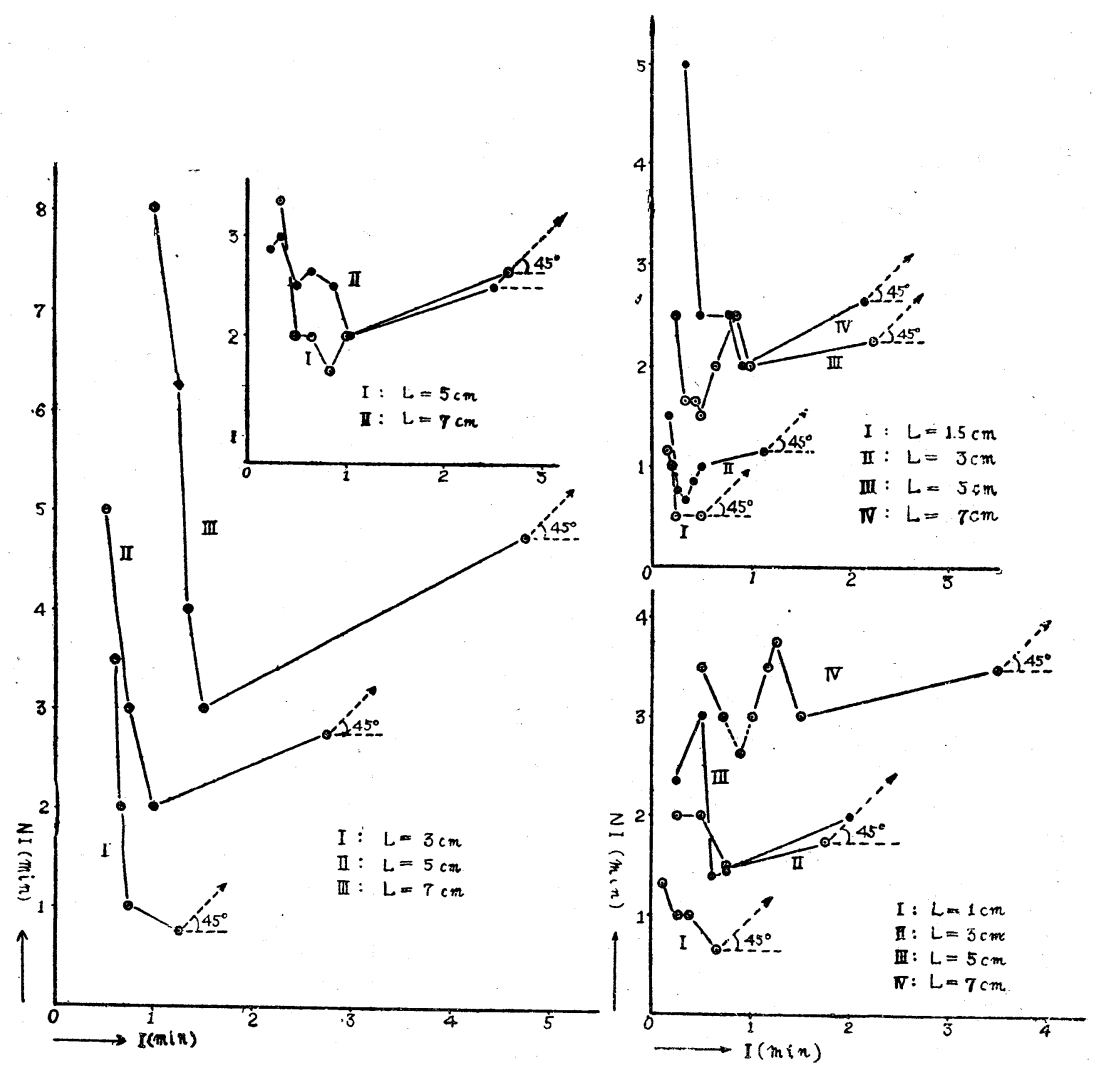

Fig. 4. $N I$ - $I$-relation in various $L$, the length of $P$-part.

\section{3. $i$ (current) $-I$ (interval)-relation}

A powerful factor which brings out the characteristic $N$-I-relation above described, should perhaps be sought in the intensity of the activation current which will vary with varying intervals. The current flowing over the $P$-part was, therefore, brought under examination. The examination was carried out in the following way (fig. 5): Instead of covering a part of the core with paraffin, the part was drawn out into the air to be connected to a milliammeter.

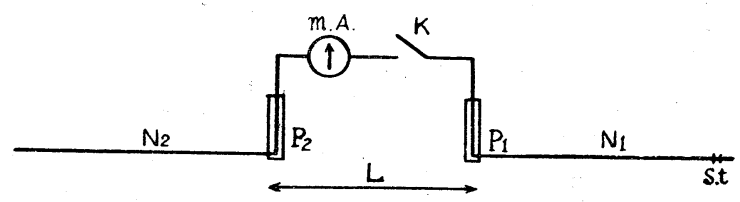

Fig. 5. Examination of the activation current.

m.A., milliammeter.

$P_{1,2}$, paraffin covers.

$L$, the distance between $P_{1}$ and $P_{2}$, corresponding to the

$K$, key. 
The two up-right parts of the model $\left(P_{1,2}\right.$ in fig. 5$)$ were covered with paraffin and placed face to face at a variable distance $L$. The milliammeter served as a measuring instrument, as, to our regret, no oscillograph was available.

Now, with $K$ closed, a $\mathrm{Zn}$-stimulus is given at the right end of $N_{1}$. If the immersion time is long and, consequently, the wave jumps over $L$ to $N_{2}$, we have a quick diphasic movement of the needle of the meter. But if the immersion time is short and the wave stops at this side of $P$, then we have a monophasic movement instead of a diphasic. In both cases, a slow and small elevation is seen appearing in the earlier phase of ascent, which bursts into a sudden and large deflection when the wave reaches $P_{1}$. It was confirmed later that the small elevation was due partly to the spread of the stimulating current, but in large part to the activation wave proceeding along $N_{1}$, because it continued to increase also after the stimulator (a $\mathrm{Zn}$ piece) was taken off.

The monophasic variation is also obtained when the post- $L$ part (that is, $N_{2}$ ) has been activated just previous to $N_{1}$. Generally, the monophasic movement is sufficiently slow (and the deflection is even larger than that of the diphasic, evidently owing to the slowness of the movement of the meter), so that we can read off the pointed value with certainty. Hence, we decided, for convenience sake, to activate first $N_{2}$, with $K$ open, exactly $5^{\prime \prime}$ prior to $N_{1}$-activation and then, with $K$ closed, to activate $N_{1}$ to measure the current due to $N_{\mathrm{I}}$-activation.

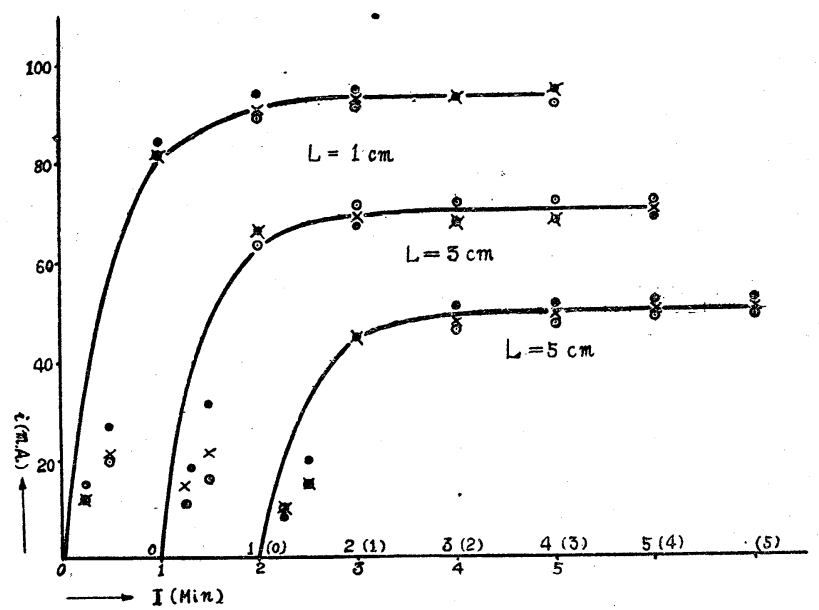

Fig. 6. $i$-I-relation, $i$ being the activation current.

The results thus obtained are illustrated in fig. 6. As seen in the figure, the strength of current rises steeply and then slowly with longer intervals, to attain the highest value in about $1 \sim 2$ minutes. The absolute values depend of course upon the length of $L$, the only variable electroresistant part.

\section{Parallel explorations of N-I-, NI-I- and i-I-relations}

The $i$ - $I$-relation described above seemed to promise an explanation for the $N$-I-relation obtained before. For this, it was planned to explore both $N$ - $I$ - and $i-I$-relations on one and the same model under one and the same condition. A 
few examples of the results obtained are shown graphically in fig. 7. The minimum point in question exists when $L$ is large (fig. $7, a$ ) and not when $L$ is small (fig. $7, c$ ), as was noticed before. And when it exists, it is found in the neighbourhood of such an interval, at which the current first attains, in approximation, its full size.

\section{CONSIDERATIONS}

\section{The minimum point observed in NI-I-curves}

The number of successive activation waves $(N)$ necessary for evoking just one transmission, increases first slowly, then steeply, with shortening the interval ( $I$ ) of activation. Of course there is, if finely examined, a certain definite range of interval for each $N$, as was described above, so that the whole $N$ - $I$-curve is not smooth but of a step-like shape, the . breadth of the step getting narrower with shorter intervals.

A point to be remarked is that there is generally a minimum point in the $N I-I$-curve, which is constructed from the $N$ - $I$-curve actually obtained. The minimum point appeared clearly (fig. $7, a$ ) in the case of large $L$, that is, in the case of greater distance over which the wave has to jump for further conduction, whereas it is absent (fig. $7, c$ ) if $L$ is small. In other words, the minimum point in question was clearly observable when the wave is facing a relatively large hindrance to get over for further transmission.

On the other hand, the current flowing in the core over $L$ was found to increase gradually with interval, the full strength being attained at intervals of about $1 \sim 2$ minutes. Now, it was found, by parallel explorations, that the minimum point above mentioned is found at the interval at which the current nearly attains its highest value. Notice, now,
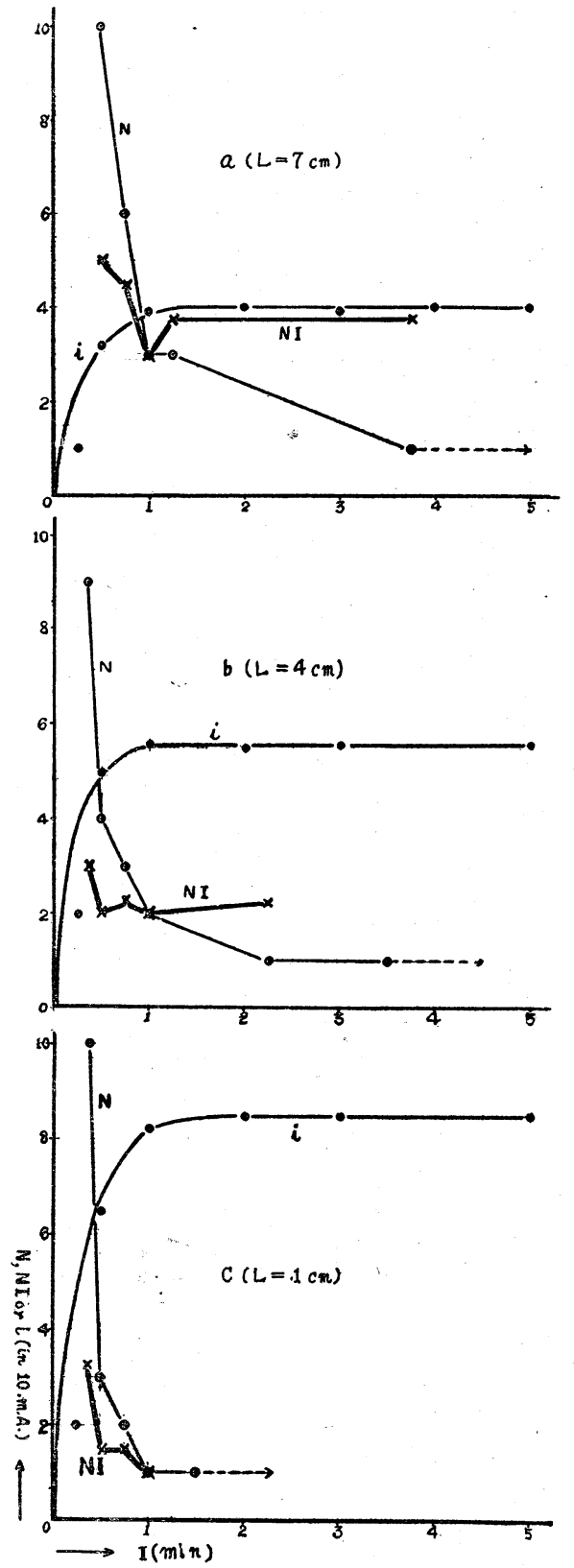

Fig. 7. $N, N I$ and $i$ related to $I$. 
that in fig. $7, a$, the minimum point lies at the $1^{\prime}$-interval, at which the current is about maximal ( $39 \mathrm{~mA}$ for $40 \mathrm{~mA}$ ). In this case, the least interval for the case $N=1$, was $3^{\prime} 45^{\prime \prime}$, and the current strength required was $40 \mathrm{~mA}$. Hence, to repeat the activation at a $1^{\prime}$-interval is to repeat the activation with a nearly maximal current intensity, and, consequently, there is a great possibility of producing a large summation effect, which, in turn, would, possibly and probably, have caused to make $N I$ smaller than $3^{\prime} 45^{\prime \prime}$.

In the case of fig. 7, $c$, however, where the minimum point is lacking, the least interval for the case $N=1$ is $1^{\prime}$, and the current required is $83 \mathrm{~mA}$, which is about the highest magnitude in this case. As seen in the figure, the current gets abruptly weaker if the interval is made shorter than $1^{\prime}$. So, a repetition of activation with intervals shorter than $1^{\prime}$ is, in contrast to the case of fig. 7, $a$, a repetition with much less effective currents, hence there will hardly be a possibility of producing a large summed effect or, in other words, of producing the minimum point in the NI-I-curve.

\section{The significance of the minimum point observed}

The existence of a minimum point in the NI-I-curve indicates that, for obtaining a transmission by successive waves, there is an optimum frequency of activation in the sense that the total time required becomes then the smallest value. The direct cause seems to be in intimate relation with the intensity variation of the activation current, in association with change of intervals.

Whether the same thing will happen in the nervous system (at, for example, the synapse) or at the junction between nerve and its effector organ, is of course an open question. The point will lie in whether there is or not a certain range of short sitmulation interval, at which either the nervous impulse itself or its effect on the effector organ will appear smaller than usual. The impulse, however, will be invariable in magnitude without regard to changing interval, while its "effect" produced in the effector organ will generally appear weaker if examined at intervals shorter than a certain limit, sometimes because of the heterobolic nature of the organ, and sometimes because of the slower temporal course of excitation and recovery in comparison to that of nerve fibres. We suppose, thus, that the condition for the occurrence of minimum NI described above is perhaps obtainable in living systems, too, if the nerve and its effector organ are taken together as a functional unit. In fact, cases are known, for instance, where a single impulse cannot pass the central region, while successive impulses can, evidently due to the relatively slow temporal course of decay of the excitatory state and, accordingly, to the relatively easy temporal summation of the excitatory effect there. Our supposition is that in such cases as these the total time-length, that is, the interval multiplied by the number of impulses required for one transmission, will perhaps assume a minimum value at a certain stimulus frequency. This supposition, if it is right, suggests that there is a proper rhythm for each nerve, and that, if the nerve is excited with this particular rhyhm, the time required for a transmission, or for producing a certain final event in general, will be economized. Experimental examinations are desirable. 
A certain length in the middle portion of a Lillie's nerve model was covered with paraffin, so as an activation wave approaching it has to jump over it for further conduction. The paraffined part was made sufficiently long, so that a single wave could first pass it when the immersion time was made correspondingly long. At a shorter immersion time, a certain number of successive waves were needed for the occurrence of one transmission.

Under such an arrangement, examinations were made on

1) $N$ - $I$-relation, where $N$ is the number of waves needed, and $I$ the stimulation interval, that is, the immersion time,

2) NI-I-relation,

3) $i$ - $I$-reration, $i$ being the current flowing over the paraffined part.

The results showed that $N$ increased inversely with $I$, while $N I$ showed a minimum point in relation to $I$. On the other hand, $i$ increased with $I$ until it attains its highest value at an interval of about $1 \sim 2$ minute.

The appearance of the minimum point was attributed to the variation of $i$ associatiated with changing $I$. It was supposed that a similar state might happen in a nerve, and that, then, the rhythm of excitation for producing the minimum $N I$ will be characteristic to each nerve, and further that if a nerve is excited with this particular rhythm, it will be significant in economizing the time for realizing the transmission.

\section{REFERENCES}

1. LAPICQUe, L. D'èxcitabilitè iterative. Première Partie. Paris: Hermann et Cie, 1936.

2. Bishop, G. H. AND Heinbecker, P. Am. J. Physiol. 100: 519, 1932.

Rosenblueth, A., Forbes, A. and Lambert, E. Am. J. Physiol. 105 : 508, 1933.

FredericQ, H. Bull. Acad. Med. Belgique. p. 450, 1934. (quoted in Lapicque's book cited above.)

3. YAMAGIW A, K. Jap. Med. J. 1: 557, 1948.

The expenses for this work were defrayed by a grant from the National Research Council of Japan. 